\title{
NOUVELle
}

\section{La capside du virus de l'immunodéficience humaine au centre d'un engouement sans précédent}

Nathalie Jane Arhel

> L'année 2021 marque le $40^{\circ}$ anniversaire de l'identification des premiers patients atteints du syndrome d'immunodéficience acquise (Sida), une infection chronique par un rétrovirus du genre lentiviridae. Il existe aujourd'hui de nombreux médicaments antiviraux ciblant différentes étapes du cycle de ce virus de l'immunodéficience humaine (VIH), et qui, combinés, permettent de réprimer de manière efficace sa réplication. La thérapie antirétrovirale garantit aujourd'hui aux patients la même espérance de vie qu'à une personne non infectée, et réduit sensiblement la transmission du virus d'une mère à son enfant, ou d'un individu infecté à son partenaire sain [1]. Dans ce contexte d'efficacité thérapeutique, on peut s'interroger sur l'intérêt des recherches intensives qui sont encore menées pour mieux comprendre ce virus, et notamment l'engouement sans précédent qu'a suscité la capside du VIH en 2020 et 2021, avec une dizaine d'articles parus dans des journaux à fort facteur d'impact [2-10].
Le besoin de nouvelles solutions thérapeutiques pour les personnes atteintes du Sida

À ce jour, environ un tiers des personnes infectées dans le monde, soit 12 millions d'individus, n'ont pas accès à une thérapie antirétrovirale (rapport 2020 d'ONUSIDA $^{1}$ ). Par ailleurs, malgré l'arsenal thérapeutique disponible, la prise quotidienne de médicaments tout au long de la vie entraîne des défauts d'observance, particulièrement chez les patients traités depuis longtemps, ou souffrant de troubles psychiques ou de toxicomanie. Le manque d'observance rigoureuse peut favoriser l'apparition de souches virales résistantes et conduire à un échec thérapeutique. Enfin, même en cas d'observance thérapeutique rigoureuse, le VIH persiste sous forme latente dans l'organisme, ce qui peut être à l'origine de maladies chroniques inflammatoires et d'un vieillissement

1 Programme commun des Nations Unies contre le VIH/Sida (http://www.unaids.org).
Institut de recherche en infectiologie de Montpellier (IRIM), CNRS UMR9004, Université de Montpellier, 1919 route de Mende, 34090 Montpellier, France. nathalie.arhel@irim.cnrs.fr

prématuré malgré l'absence de charge virale détectable. Ainsi, le développement d'antiviraux plus efficaces, à longue durée d'action, voire curatifs, reste un objectif prioritaire de santé publique $[11,12]$.

\section{La capside du VIH}

Le VIH, comme tous les virus, est composé d'un génome entouré d'une capside. Les capsides virales sont des assemblages protéiques qui, selon les virus, peuvent comporter différentes symétries, telles qu'une symétrie icosaédrique (herpesviridae, flavivirae) ou une symétrie hélicoïdale (virus de la grippe). La capside du VIH est conique et mesure environ $120 \mathrm{~nm}$ en longueur et 40 à 60 nm en largeur. Elle est composée d'environ 2500 monomères de la protéine p24, appelés CA (capside), qui forment des dimères puis s'assemblent pour former plus de 200 hexamères et 12 pentamères (Figure 1) [13]. La protéine CA est la plus conservée des protéines du VIH, et joue un rôle essentiel dans 


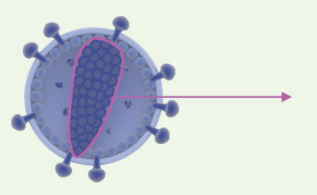

Virion VIH

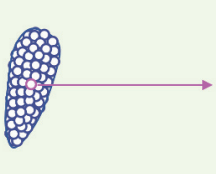

Capside du VIH
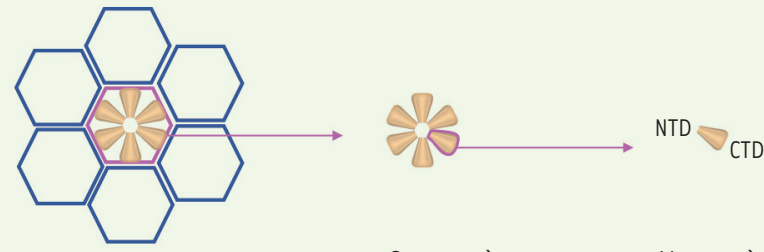

Capsomère Assemblage hexagonal (hexamère ou pentamère)
Monomère

(CA/p24)

Figure 1. Structure de la capside du VIH. La capside du VIH, qui entoure le génome viral, est composée de plusieurs milliers de monomères (CA/ p24), qui s'assemblent grâce à leurs domaines $N$ - et C-terminaux (NTD et CTD, respectivement) pour former des hexamères ou des pentamères. Ces capsomères s'assemblent à leur tour pour former la surface incurvée de la capside conique.

plusieurs étapes du cycle, notamment l'assemblage, la rétro-transcription, le transport intracellulaire, l'entrée dans le noyau et l'intégration du génome viral à I'ADN cellulaire $[14,15]$. La capside du VIH interagit, via l'interface incurvée de l'assemblage hexamérique, avec de nombreux partenaires cellulaires qui contrôlent sa stabilité (CypA, PDZ8, IP6, (PSF6), son déplacement dans la cellule (MAP1, BicD2, RanBP2, Nup153) ou ses cinétiques de désassemblage (TRIM $5 \alpha$, TRN 1, TRIM11, TRIM34, TRIMCyp, MxB, Daxx, Pinl, MELK, KIF5B). La stabilité de la capside est essentielle à l'infectivité du virus, et un assemblage trop stable ou trop fragile réduit son pouvoir infectieux.

\section{Le potentiel thérapeutique}

de la capside du VIH

La capside du VIH-l est considérée depuis longtemps comme une cible thérapeutique attractive, et plusieurs inhibiteurs ciblant la protéine CA ont déjà été décrits, comme par exemple CAP-1, PF-3450074 (PF74), GS-CAl et GS-6207 [16]. Des études récentes ont montré que ces inhibiteurs se démarquent sensiblement des autres antirétroviraux actuellement approuvés pour le traitement des patients. En effet, contrairement aux autres molécules antirétrovirales, qui ciblent des étapes précises du cycle de réplication virale (entrée, rétrotranscription, intégration, ou maturation des particules virales), les inhibiteurs de capside ciblent de multiples étapes de ce cycle, en raison du rôle essentiel joué par cette capside dans les phases aussi bien précoces que tardives [14]. En particulier, GS-CAl et son dérivé GS-6207 ont un puissant effet antiviral ainsi qu'un profil d'action pléiotropique et dépendant de la dose $[2,3,17]$. Ces molécules reconnaissent l'interface hydrophobe formée par deux monomères adjacents de CA et perturbent la stabilité de la capside virale aussi bien pendant les étapes précoces, lorsque la capside doit se désassembler pour libérer le génome viral, que pendant les étapes tardives, lorsqu'elle doit à nouveau s'assembler pour produire des néo-particules. Toutefois, leur effet antiviral pléiotrope est principalement attribuable à l'inhibition des étapes précoces du cycle, où elles entraînent une stabilisation délétère de la capside virale, interfèrent avec sa capacité à interagir avec des partenaires cellulaires clés, tels que CPSF6 et Nup153, et bloquent, par deux mécanismes distincts, à la fois le transfert nucléaire du virus et l'intégration du génome viral $[2,3,17]$. Autre particularité, les inhibiteurs de capside ont des mécanismes antiviraux distincts selon la concentration utilisée [16]. Le large profil d'action des inhibiteurs de capside, leur forte stabilité métabolique et leur faible solubilité en font des candidats potentiels pour une administration par injection souscutanée conduisant à un effet persistant.

\section{De remarquables avancées dans notre compréhension de la décapsidation du $\mathrm{VIH}$}

Deux études structurales parues en 2020 ont permis d'explorer les effets pléiotropes intrigants des molécules théra- peutiques ciblant la capside du VIH-l. D'une part, de nouvelles approches de cryo-microscopie électronique à hautdébit, permettant de résoudre la structure d'assemblages multiprotéiques particulièrement flexibles et pléomorphes tels que la capside virale, ont montré que l'hexamère de CA était intrinsèquement flexible et asymétrique, apportant ainsi des données structurales solides permettant d'expliquer la différence de mécanisme d'action des inhibiteurs de capside selon les concentrations utilisées [4]. D’autre part, les processus de rétro-transcription, décapsidation et intégration du génome viral ont été reconstitués entièrement in vitro, ce qui a permis de montrer que la décapsidation n'est pas un évènement se résumant par un tout-ou-rien, mais un continuum de désassemblage [5] (Figure 2).

Les cinétiques de décapsidation du VIH dans les cellules infectées ont longtemps intrigué les chercheurs [14, 18]. Après 25 années durant lesquelles il était communément admis que la capside était dégradée dès l'entrée du virus dans la cellule, ces 15 dernières années ont vu la publication des résultats de nombreux travaux qui montraient que la capside joue un rôle essentiel dans plusieurs étapes du cycle réplicatif, notamment le transport dans la cellule, la rétro-transcription, la protection et la dissimulation du génome viral, ainsi que l'entrée dans le noyau. Ainsi, il est désormais admis que la capside du VIH est encore en grande partie intacte 


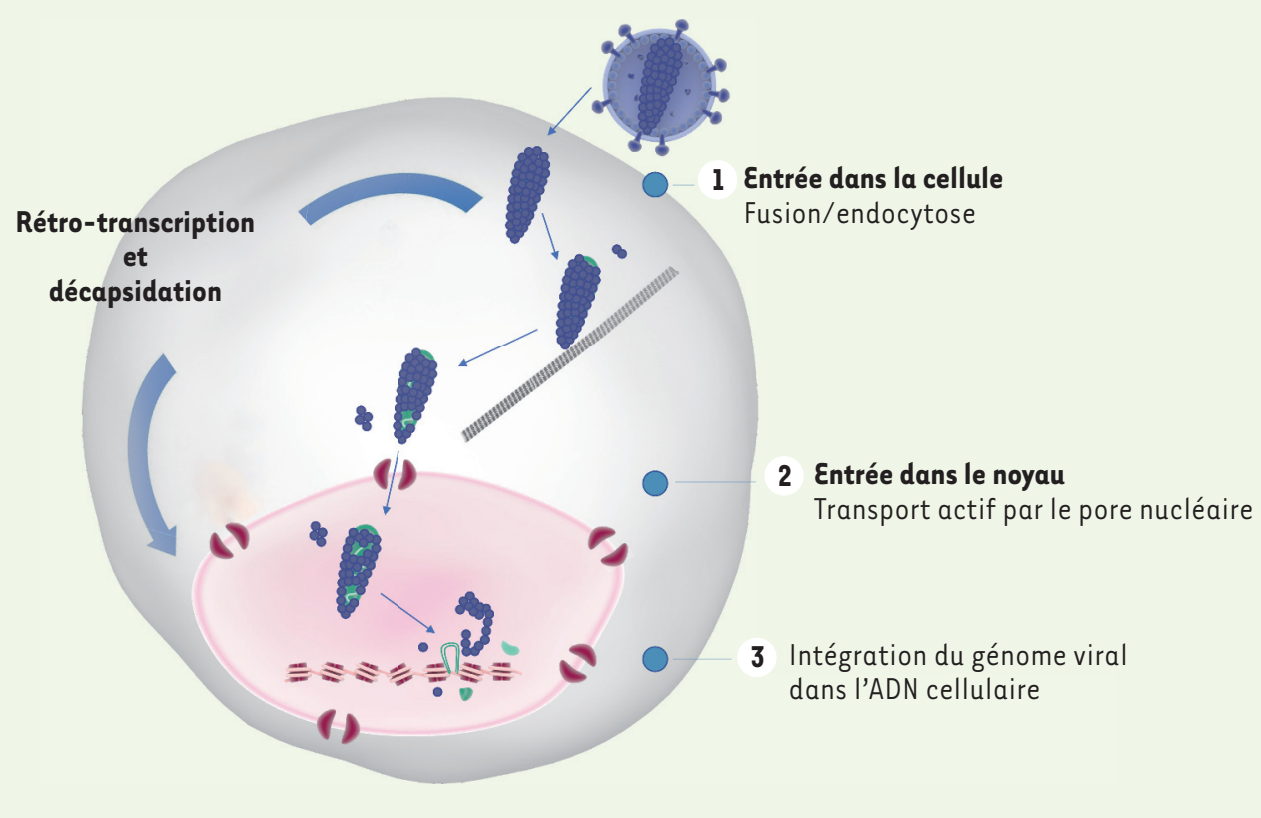

Figure 2. Représentation schématique des étapes précoces de l'infection d'une cellule par le VIH. Les résultats des travaux parus en 2020 montrent que la capside du VIH se désassemble progressivement depuis son entrée dans la cellule jusqu'à l'intégration du génome viral dans l'ADN cellulaire, et de manière concomitante au transport vers le noyau et à la rétro-transcription.

à l'entrée du pore nucléaire. Même si des études indiquaient que la protéine CA contient un signal de localisation nucléaire permettant son transport dans le noyau [19], et qu'elle participe à l'intégration du génome viral dans I'ADN de la cellule infectée [20], il était couramment admis qu'il s'agissait probablement de petits polymères de CA et non de la capside entière, dont les dimensions excèdent celles du pore nucléaire.

Toutefois, en 2020, les résultats de plusieurs études ont montré que les processus de rétro-transcription et de décapsidation pouvaient avoir lieu dans le compartiment nucléaire [6-9]. On a pu notamment observer, dans le noyau des cellules infectées, à proximité du site d'intégration du génome viral dans la chromatine, des capsides dont la conformation ressemblait à celle des virions matures $[6,21]$. Ces résultats n'excluent pas la possibilité que la rétro-transcription et la décapsidation puissent avoir lieu exclusivement dans le cytoplasme, puisque, par ailleurs, le seul prérequis à l'intégration du génome viral in vitro est la présence de molécules polyphosphorylées telles que les désoxynucléotides triphosphates (dNTP) [5, 7]. Néanmoins, ils suggèrent que l'intégration optimale du génome viral est dépendante d'une décapsidation finale dans le noyau (Figure 2).

Les résultats des recherches les plus récentes suggèrent donc que la capside du VIH se désassemble de manière progressive et continue depuis l'entrée du virus dans la cellule jusqu'à l'intégration du génome viral dans la chromatine cellulaire. II ne faut plus concevoir la décapsidation comme la dissolution d'un complexe multi-protéique en milliers de sous-unités de CA individuelles, mais plutôt comme une coque qui se fissure et s'ouvre dans des zones plus fragiles, pouvant abandonner des pans entiers de capside durant son transport vers le noyau [5]. Le paradoxe, jusqu'alors inexpliqué, du passage d'une capside virale de 40-60 nm de largeur par la lumière d'un pore nucléaire de diamètre inférieur à $30 \mathrm{~nm}$ est résolu depuis que des études structurales ont montré que ces ruptures locales permettaient à la capside de se déformer afin de surmonter la constriction radiale du pore nucléaire [5]. D'autres résultats, en revanche, suggèrent que le pore nucléaire se dilate afin de permettre aux capsides intactes de passer dans le noyau [21].

La capside virale est désormais considérée comme un élément essentiel et incontournable de la réplication du VIH, participant à de multiples étapes, précoces et tardives, y compris à l'intégration du génome viral dans I'ADN cellulaire. Une meilleure compréhension de la contribution de la capside virale à ces différentes étapes est nécessaire à l'aboutissement de la recherche d'une nouvelle classe de molécules antivirales. $\diamond$

An unprecedented interest for the human immunodeficiency virus capsid

\section{REMERCIEMENTS}

N.J. Arhel est employée par le CNRS.

\section{LIENS D'INTÉRÊT}

L'auteure déclare n'avoir aucun lien d'intérêt concernant les données publiées dans cet article. 


\section{RÉFÉRENCES}

1. Ghosn J, Taiwo B, Seedat S, et al. HIV. Lancet 2018 ; $392: 685-97$.

2. Bester SM, Wei G, Zhao H, et al. Structural and mechanistic bases for a potent HIV-1 capsid inhibitor. Science $2020 ; 370: 360-4$.

3. Link J0, Rhee MS, Tse WC, et al. Clinical targeting of HIV capsid protein with a long-acting small molecule. Nature 2020 ; 584 : 614-8.

4. $\mathrm{Ni}$ T, Gerard S, Zhao G, et al. Intrinsic curvature of the HIV-1 CA hexamer underlies capsid topology and interaction with cyclophilin A. Nat Struct Mol Biol 2020 ; $27: 855-62$.

5. Christensen DE, Ganser-Pornillos BK, Johnson JS, et al. Reconstitution and visualization of HIV-1 capsiddependent replication and integration in vitro. Science $2020 ; 370$.

6. Burdick RC, Li C, Munshi M, et al. HIV-1 uncoats in the nucleus near sites of integration. Proc Natl Acad Sci USA 2020 ; 117 : 5486-93.

7. Dharan A, Bachmann N, Talley S, et al. Nuclear pore blockade reveals that HIV-l completes reverse transcription and uncoating in the nucleus. Nat Microbiol 2020 ; 5 : 1088-95.
8. Selyutina A, Persaud M, Lee K, et al. Nuclear import of the HIV-1 core precedes reverse transcription and uncoating. Cell Rep 2020 ; 32 : 108201.

9. Rensen $\varepsilon$, Mueller F, Scoca V, et al. Clustering and reverse transcription of HIV-l genomes in nuclear niches of macrophages. EMBO J 2021 ; 40 : e105247.

10. Müller TG, Zila V, Peters K, et al. HIV-l uncoating by release of viral cDNA from capsid-like structures in the nucleus of infected cells. bioRxiv 2021 (soumis pour publication).

11. Ndung'u T, McCune JM, Deeks SG. Why and where an HIV cure is needed and how it might be achieved. Nature 2019 ; 576 : 397-405.

12. Nyaku AN, Kelly SG, Taiwo BO. Long-acting antiretrovirals: Where are we now? Curr HIV/AIDS Rep 2017 ; $14: 63-71$.

13. Mattei S, Glass B, Hagen WJ, et al. The structure and flexibility of conical HIV-1 capsids determined within intact virions. Science 2016 ; 354 : 1434-7.

14. Campbell EM, Hope TJ. HIV-1 capsid: the multifaceted key player in HIV-1 infection. Nat Rev Microbiol 2015 ; $13: 471-83$.

15. James $L C$, Jacques DA. The human immunodeficiency virus capsid is more than just a genome package. Annu Rev Virol 2018 ; 5 : 209-25.
16. Carnes SK, Sheehan JH, Aiken C. Inhibitors of the HIV-1 capsid, a target of opportunity. Curr Opin HIV AIDS 2018; 13 : 359-65.

17. Yant SR, Mulato A, Hansen D, et al. A highly potent long-acting small-molecule HIV-l capsid inhibitor with efficacy in a humanized mouse model. Nat Med $2019 ; 25: 1377-84$.

18. Arhel N. Revisiting HIV-1 uncoating. Retrovirology $2010 ; 7: 96$.

19. Fernandez J, Machado AK, Lyonnais S, et al. Transportin- 1 binds to the HIV-l capsid via a nuclear localization signal and triggers uncoating. Nat Microbiol $2019 ; 4$ : 1840-50.

20. Engelman AN, Singh PK. Cellular and molecular mechanisms of HIV-1 integration targeting. Cell Mol Life Sci $2018 ; 75$ : 2491-507.

21. Zila V, Margiotta $\varepsilon$, Turonova B, et al. Cone-shaped HIV-l capsids are transported through intact nuclear pores. Cell 2021 ; 184 : 1032-46.

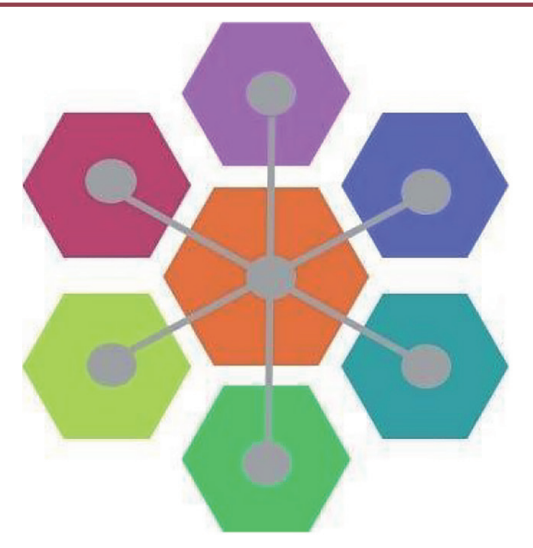

\section{Globall Registry for COL6-related dystrophies}

Registre global des dystrophies liées au collagène de type VI

S'inscrire sur : www.collagen6.org

Ou contactez-nous par e-mail à l'adresse : collagenbregistry@ncl.ac.uk

La traduction française sera bientôt disponible sur le site web.
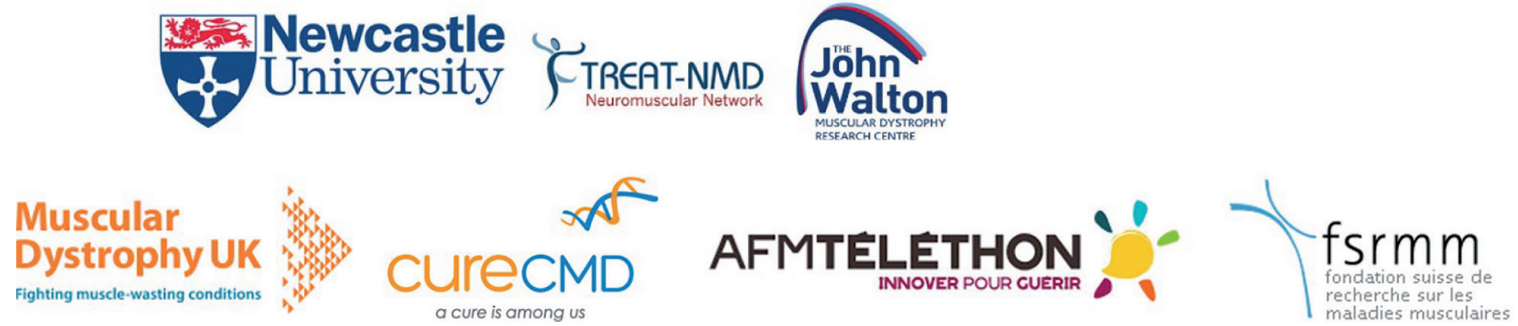

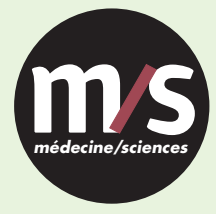

Tarifs d'abonnement $\mathrm{m} / \mathrm{s}-2021$

Abonnez-vous

à médecine/sciences
$>$ Grâce à $m / s$, vivez en direct les progrès des sciences biologiques et médicales

Bulletin d'abonnement page 554 dans ce numéro de $\mathrm{m} / \mathrm{s}$

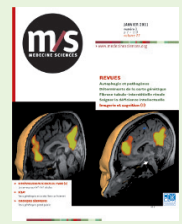

